was found for intermediate levels of glucose intolerance, although the study was underpowered to assess this association.

Conclusions: Irrespective of the exposure measure and the confounders controlled for, diabetes was consistently found to be associated with an increased risk of TB. This study may underestimate the true association between the two diseases, due mainly to exposure misclassification, as only $24.8 \%$ of the sample took the OGTT. Due to the inclusion of "ever diagnosed" as opposed to incident TB cases, the direction of the association could not be reliably assessed and may operate in both directions. Some unmeasured factors may have attenuated or increased the relationship, although the majority of known confounders were controlled for. These results may be more generalisable to low TB prevalence populations than to populations where $\mathrm{TB}$ is endemic.

\section{PREVALENCE OF CHRONIC KIDNEY DISEASE IN SOUTH ASIAN AND BLACK MINORITIES: FINDINGS FROM A POPULATION BASED SCREENING STUDY IN LONDON, UK}

${ }^{1} \mathrm{P}$ Roderick, ${ }^{2} \mathrm{~L}$ Lightstone, ${ }^{1} \mathrm{~S}$ Harris, ${ }^{3} \mathrm{M}$ Lapsley, ${ }^{3} \mathrm{M}$ Dockrell, ${ }^{2} \mathrm{E}$ Lim, ${ }^{2} \mathrm{~J}$ Chambers, ${ }^{2} J S$ Kooner. ${ }^{1}$ Public Health Sciences and Medical Statistics, University of Southampton, Southampton, UK; ${ }^{2}$ mmperial College London, London, UK; ${ }^{3}$ South West Thames Kidney Institute, St Helier's Hospital, London, UK

\section{doi:10.1136/jech.2009.096727|}

Background: People of both South Asian and Black ethnic origin have 3-4 fold higher rates of acceptance onto renal replacement therapy than Caucasians in the UK. They are known to have a higher prevalence of type 2 diabetes and Blacks also have a higher prevalence of hypertension but little is known about the comparative prevalence of chronic kidney disease (CKD) in these ethnic groups.

Objective: To investigate the prevalence of CKD in Blacks and south Asians compared to Caucasians.

Methods: Cross sectional study based on screening all adults registered with over 50 general practices aged 35-74 in a multiethnic area in West London, UK from 2002-7. Baseline assessment included renal function (serum creatinine converted to estimated glomerular filtration rate using 4 variable MDRD equations) and single urinary albumin: creatinine ratio (ACR) from 2004. Logistic regression was used to model adjusted odds ratios of low eGFR $\left(<60\right.$ and $<45 \mathrm{ml} / \mathrm{min} / 1.73 \mathrm{~m}^{2}$ ) indicative of stage 3-5 and stage 3b-5 CKD respectively, by ethnic group separately by gender, with Caucasians as reference group.

Results: Response rate to screening was 60\%. Of 31507 participants, 19769 (63\%) were South Asian (SA), 9222 (29\%) Caucasian and 2516 (8\%) Black (B). All had eGFR calculable. Age adjusted prevalence of eGFR $<60$ was 1.29 (1.10 to 1.51) in SA males, 0.87 (0.62 to 1.23 ) in B males, 0.74 (0.63 to 0.89 ) in SA females and 0.35 (0.23 to 0.54$)$ in $B$ females. Corresponding figures for eGFR $<45$ were SA males 1.89 (1.38 to 2.58) B males 1.64 (0.93 to 2.89) SA females 0.92 (0.56 to 1.34) and B females 0.50 (0.21 to 1.18). Full adjustment for prevalent diabetes, hypertension, vascular disease and social deprivation did not alter these patterns.

Conclusion: Despite a higher prevalence of underlying risk factors such as Type 2 diabetes, CKD prevalence of stage $3-5($ eGFR $<60)$ was generally lower in both ethnic groups except South Asian males. However more advanced CKD $(e G F R<45)$ in men was commoner in both ethnic groups suggesting they are more susceptible to progressive kidney damage. Urinary albumin excretion, an important marker of kidney damage, is being measured currently and data will be presented. The reasons for the gender difference in kidney function require further exploration.

\section{Disability and capability}

\section{THE ASSOCIATION BETWEEN PARTICIPATION OF CHILDREN WITH CEREBRAL PALSY AND THE PHYSICAL, SOCIAL AND ATTITUDINAL ENVIRONMENT: A CROSS-SECTIONAL EUROPEAN STUDY}

HO Dickinson, A Colver. Institute of Health and Society, Newcastle University, Newcastle upon Tyne, UK

doi:10.1136/jech.2009.096727m

Background: Both the UN Convention on the Rights of the Child and the UN Convention on the Rights of Persons with Disabilities affirm the right of children with disabilities to participate on an equal basis with others in family life, health maintenance, education, public life, recreational, leisure and sporting activities.

Objective: To assess, for children with cerebral palsy, the extent of availability of needed items in the physical, social and attitudinal environment and to evaluate how this is associated with the children's participation in life situations.

Design: Following preliminary qualitative studies, the European Child Environment Questionnaire (ECEQ) was developed to record which items in the physical, social, and attitudinal environment of home, school, and community are available to children with disabilities. The ECEQ was administered to parents of children with cerebral palsy. Children's participation was assessed using the Life-H questionnaire. The 60 items of ECEQ were grouped into domains using item response models. Structural equation modelling was used to relate the child's participation to environmental factors, allowing for impairments, pain and socio-demographic characteristics.

Setting: Eight European regions with population registers of children with cerebral palsy; one further region recruited children from multiple sources.

Participants: 1174 children with cerebral palsy aged 8-12 years randomly selected from the population registers, 743 (63\%) agreed to joined in the study; the further region recruited 75 children.

Main Outcome Measures: Children's participation, assessed on 10 domains of the Life-H questionnaire.

Results: Children with pain and those with more severely impaired walking, fine motor skills, communication and intellectual abilities had lower participation across most domains, but the sociodemographic factors examined were not associated with participation. We identified nine domains describing the accessibility of the environment. All domains of both participation and environment showed significant $(p<0.001)$ variation between regions. Results of the structural equation modelling will be presented.

Conclusions: Some European regions facilitate participation of children with cerebral palsy better than others and some regions have a more accessible environment than others, implying some countries could improve provision.

\section{TWENTY-YEAR SURVIVAL OF CHILDREN BORN WITH CONGENITAL ANOMALIES: A POPULATION-BASED STUDY}

${ }^{1} \mathrm{P}$ Tennant, ${ }^{1} \mathrm{M}$ Pearce, ${ }^{2} \mathrm{M}$ Bythell, ${ }^{1,2} \mathrm{~J}$ Rankin. ${ }^{1}$ Institute of Health and Society, Newcastle University, Newcastle upon Tyne, UK; ${ }^{2}$ Regional Maternity Survey Office, Newcastle upon Tyne, UK

doi:10.1136/jech.2009.096727n

Objective: To estimate the survival, up to age 20 years, for a range of congenital anomaly groups and subtypes.

Design: Population-based registry (Northern Congenital Abnormality Survey, NorCAS).

Setting: The former Northern Region of England (the area extending from North Cumbria to the Tees area and up to the 
Scottish border), a geographically distinct area with a stable population of three million, and approximately 35000 deliveries per year.

Participants: 13738 individuals with at least one congenital anomaly (classified according to the European Surveillance of Congenital Anomalies, EUROCAT, guidelines) born between 01 January 1985 and 31 December 2003, of whom 10951 were live born and 10837 (99\%) were traced for their survival status on 28 January 2008.

Main Outcome Measures: Congenital anomaly group and subtype specific survival rates at one week, four weeks, one year, five years, 10 years, 15 years and 20 years. Change in survival, and the number of terminations of pregnancy for fetal anomaly, with time.

Results: Survival among children with at least one congenital anomaly was $88.8 \%$ (95\% CI 88.2 to 89.4$)$ and 85.5 (95\% CI 84.8 to $86.3)$ at one year and 20 years respectively. Twenty year survival among the most common anomaly groups was $89.5 \%$ (95\% CI 88.4 to 90.6 ) for cardiovascular, $79.1 \%$ (95\% CI 76.7 to 81.3 ) for chromosomal, $93.2 \%$ (95\% CI 91.6 to 94.5$)$ for urinary, $83.1 \%(95 \%$ CI 79.6 to 86.0 ) for digestive system, $97.7 \%$ (95\% CI 96.1 to 98.7 ) for orofacial clefts, and $66.2 \%$ (95\% CI 61.5 to 70.4) for nervous system anomalies. Survival varied considerably between subtypes, even within the same anomaly group. The proportion of terminations for fetal anomaly increased throughout the study period, and, together with year of birth, was an independent predictor of survival.

Conclusions: This study presents robust estimates of survival, uniquely to age 20 years, for a range of congenital anomaly groups and subtypes, some of which have been infrequently reported previously. This information will be valuable for families, genetic councillors and other health care professionals when a congenital anomaly is detected, and will assist in planning for the future care needs of affected individuals.

\section{MEASURING LOCOMOTOR DISABILITY: A NEW SCORING MECHANISM FOR SOME FAMILIAR ITEMS}

S Muller, E Thomas, G Peat. Arthritis Research Campaign National Primary Care Centre, Primary Care Sciences, Keele University, Keele, Staffordshire, UK

\section{doi:10.1136/jech.2009.0967270}

Background: Locomotor disability is a critical measure for studying functional decline in later life and has featured in several major epidemiological studies. Yet measurement has mostly been confined to single items or ordinal scales rather than truly unidimensional measures with interval properties, thereby limiting the detection and interpretation of change in locomotor disability over time. Our aim was to combine items from the well-known SF-36 Physical Functioning Scale (PF-10) into a single score using Rasch analysis and test the properties of this new measure.

Methods: A health survey was sent to a random sample of 500 adults aged 50 years and over registered with a single general practice in North Staffordshire. The questionnaire contained the PF10 , from which five items (two relating to stair climbing and three to walking) were selected. Response dependency exists between the items, and so they were formed into two "super-items" (stairclimbing and walking). These items were assessed for their fit to the Rasch measurement model in terms of threshold ordering, unidimensionality, item and person fit, invariance along the latent trait and differential item functioning (DIF) by age-group (50-65, $66+$ years) and gender. Rasch analysis was carried out in RUMM2020. The score was confirmed in three external datasets and tests carried out for its repeatability and validity.

Results: 372 people responded to the health survey and of these, 368 responded to at least one of the five PF-10 items. Both superitems displayed disordered thresholds that were corrected by combining adjacent response categories. There was no evidence against unidimensionality, and item and person fit was good. There was no evidence against invariance along the latent trait and no signs of DIF by either age-group or gender. The items from the external datasets also met Rasch model specifications and the score was shown to be repeatable and valid.

Conclusions: Applications of measurement scales with confirmed interval level properties are advantageous for epidemiological studies using repeated measures over time. We have developed and externally validated a scoring method for deriving a brief selfreported locomotor disability scale with Rasch properties from commonly gathered items. This should allow the interval-level measurement of locomotor disability in general populations and so improve the quality of longitudinal studies in this area. The use of items from the PF-10 means that this improvement in quality is at no extra burden to the respondent or researcher, as in many cases, data can be analysed retrospectively.

\section{PHYSICAL CAPABILITY AND SUBSEQUENT MORTALITY: A SYSTEMATIC REVIEW AND META-ANALYSIS OF PUBLISHED DATA}

R Cooper, D Kuh, R Hardy, the FALCon study team. MRC Unit for Lifelong Health and Ageing, Department of Epidemiology and Public Health, Royal Free and University College Medical School, London, UK

doi:10.1136/jech.2009.096727p

Background: Evidence suggests that measures of physical capability may be predictive of subsequent disability, health and mortality, but the results from existing published studies have not been synthesised.

Objectives: To review published literature examining the associations between physical capability and subsequent all-cause mortality.

Design: Systematic review and meta-analysis.

Sources: Medline (from 1950 to 2008/05), EMBASE (from 1980 to 2008/02) and manual searches of the reference lists of eligible papers.

Included Studies: Any study examining the association between at least one of the specified measures of physical capability (grip strength, chair rises, standing balance, walking speed/time) and mortality in a population who were healthy and communitydwelling at baseline.

Results: Of 2240 unique records screened, 20 papers met the inclusion criteria of the review. An additional 3 papers were identified from reference lists. Within the 23 papers, there were 17 published analyses of grip strength, 9 of walking time/speed, 2 of standing balance and 1 of chair rises. The mean age of participants ranged from 36 to 80 y but the majority of study populations were aged $60 \mathrm{y}$ and above. Of the papers investigating the association between grip strength and mortality, 6 presented Hazard Ratios for a unit change in grip strength with basic adjustments (total $\mathrm{n}=10171$, deaths $=4267$ ). The pooled Hazard Ratio for mortality per $5 \mathrm{~kg}$ increase in grip strength, from a random effects meta-analysis, was 0.90 (95\% CI 0.87 to 0.93). Heterogeneity between studies $\left(\mathrm{I}^{2}=65.2 \%\right)$ was largely explained by a single study which had weaker results. The pooled HR when this study was removed was 0.89 (0.86 to 0.92$)\left(\mathrm{I}^{2}=25.7 \%\right)$. Other papers, where comparisons between categories of grip strength had been performed, also generally found weaker grip strength to be associated with higher rates of mortality but these analyses were too heterogeneous to be combined using meta-analysis. Due to differences in the ways in which walking speed had been measured and analysed it was not possible to perform meta-analyses of these data, but the results from all 9 studies showed that slower walking speed/longer walking time was significantly associated with increased mortality risk.

Conclusions: This review demonstrates that grip strength and walking time/speed are useful markers of subsequent mortality risk in community-dwelling populations. The underlying explanations 